\title{
ARBITRARILY SLOW APPROACH TO LIMITING BEHAVIOR
}

\author{
K. GOLDEN AND S. GOLDSTEIN
}

(Communicated by R. Daniel Mauldin)

\begin{abstract}
Let $f(\mathbf{k}, t): \mathbb{R}^{N} \times[0, \infty) \rightarrow \mathbb{R}$ be jointly continuous in $\mathbf{k}$ and $t$, with $\lim _{t \rightarrow \infty} f(\mathbf{k}, t)=F(\mathbf{k})$ discontinuous for a dense set of $\mathbf{k}$ 's. It is proven that there exists a dense set $\Gamma$ of $\mathbf{k}$ 's such that, for $\mathbf{k} \in \Gamma,|f(\mathbf{k}, t)-F(\mathbf{k})|$ approaches 0 arbitrarily slowly, i.e., roughly speaking, more slowly than any expressible function $g(t) \rightarrow 0$. This result is applied to diffusion and conduction in quasiperiodic media and yields arbitrarily slow approaches to limiting behavior as time or volume becomes infinite. Such a slow approach is in marked contrast to the power laws widely found for random media, and, in fact, implies that there is no law whatsoever governing the asymptotics.
\end{abstract}

\section{INTRODUCTION}

Many systems exhibit a well-defined limiting behavior as time or volume becomes infinite. It is often difficult, however, to obtain rigorous information on the rate of approach to the limiting behavior, which is of much physical interest. Here we prove a very general (superficially paradoxical) result which yields situations under which this approach is arbitrarily slow, i.e., so slow that it cannot be described by any law, be it algebraic, logarithmic, or any other. The key ingredient for such behavior is that the infinite time or volume limit depends discontinuously on some parameter $k$ which characterizes the microstructure of the system and that the discontinuities are dense. We then produce a dense set of k's for which the approach is arbitrarily slow.

The result described above is applied to diffusion and conduction in quasiperiodic media, which exhibit well-defined limiting behavior. For example, diffusion $\underline{X}_{t}$ in a quasiperiodic potential $V(\underline{x}), \underline{x} \in \mathbb{R}^{d}$, behaves on a macroscopic scale $\left(\lim _{\varepsilon \rightarrow 0} \varepsilon \underline{X}_{t / \varepsilon^{2}}\right)$ like Brownian motion with some effective diffusion tensor $\mathbf{D}^{*}(V)$ [1-3]. We analyze $\mathscr{D}(t)=E\left[\underline{X}_{t}^{2}\right] / t$ and associated functions as $t \rightarrow \infty$, where $E$ denotes averaging over diffusion paths and the phase in the potential (see $\S 3$ ) with $\lim _{t \rightarrow \infty} \mathscr{D}(t)=D^{*}=\operatorname{tr}\left(\mathbf{D}^{*}\right)$. In the case of conduction, the conductivity tensor $\boldsymbol{\sigma}^{*}(L)$ of a cubic sample of size $L$ of a medium

Received by the editors October 13, 1989 and, in revised form, February 23, 1990.

1980 Mathematics Subject Classification (1985 Revision). Primary 26A12; Secondary 60H10.

The first author's work was supported in part by NSF and AFOSR through Grants DMS-8801673 and AFOSR-90-0203.

The second author's work was supported in part by NSF Grant DMS-8903047. 
with quasiperiodic local conductivity $\sigma(\underline{x})$ converges as $L \rightarrow \infty$ to some effective conductivity tensor $\sigma^{*}$ [4], and we analyze $\sigma^{*}(L)$ as $L \rightarrow \infty$. The required discontinuity in the infinite time or volume limit is provided by the discontinuous dependence of $\mathbf{D}^{*}$ or $\sigma^{*}$ on the wavelengths of $V$ or $\sigma$, which was observed in [5]. For example, with $V(x)=\cos x+\cos k x$ in $d=1$, $D^{*}(k)$ has the same value $\bar{D}$ for all irrational $k$, but differs from $\bar{D}$ and depends on $k$ for $k$ rational, where it is thus discontinuous. (In fact, $D^{*}(k)$ is continuous at irrational $k$.) Applying our general result about approach to limiting behavior, we prove, for example, that when $V(x)=\cos x+\cos k x$, there is a dense set $\Gamma$ such that for each $k \in \Gamma,\left|\mathscr{D}(k, t)-D^{*}(k)\right|$, roughly speaking, approaches zero as $t \rightarrow \infty$ more slowly than any positive function $g(t) \rightarrow 0$ which can be explicitly written down (is expressible). For example, for $k \in \Gamma,\left|\mathscr{D}(k, t)-D^{*}(k)\right| \rightarrow 0$ more slowly than $1 / \log \cdots \log t$, for any fixed number of iterations of the logarithm. (Note that the $k$ 's in $\Gamma$ are not expressible.) As a consequence, the associated "velocity" autocorrelation function (VAF) $c(t)=E\left[\nabla V\left(\underline{X}_{t}\right) \cdot \nabla V\left(\underline{X}_{0}\right)\right]$ decays to 0 as $t \rightarrow \infty$ more slowly than any positive, expressible function integrable on $[0, \infty]$, such as $1 / t^{1+\varepsilon}$, for any $\varepsilon>0$. The Laplace transform of the VAF corresponds to the frequency$(\omega$-) dependent effective diffusivity $D(\omega)$ of the medium, with static value $D^{*}=D(0)$. For $k \in \Gamma,\left|\widetilde{D}(k, \omega)-D^{*}(k)\right|$ approaches 0 as $\omega \rightarrow 0$ more slowly than any positive expressible function of $\omega$ with limit 0 as $\omega \rightarrow 0$. For the $L$-dependent conductivity $\sigma^{*}(L)$ of a quasiperiodic medium, we obtain a similar result about $\left|\boldsymbol{\sigma}^{*}(L, k)-\boldsymbol{\sigma}^{*}(k)\right|$ as $L \rightarrow \infty$.

The arbitrarily slow approach that we obtain greatly contrasts with the rates of approach that have been previously obtained. It has been observed for particle motion in a variety of random systems [6-11] that the relevant VAF exhibits a power-law long time tail. For example [9], it is believed that the VAF for diffusion in stationary random media in $\mathbb{R}^{d}$ decays in time like $t^{-(1+d / 2)}$ as $t \rightarrow \infty$, while $D(\omega)$ approaches its static value $D^{*}$ like $\omega^{d / 2}$. Our results demonstrate that, for diffusion in quasiperiodic media, the decay of these functions obeys no such universal law, indeed, no law whatsoever.

In addition to the classical transport phenomena considered here, there has been much recent interest in quantum transport in quasiperiodic potentials [12, 13]. It is found there that the nature of the wave functions satisfying the time dependent Schrödinger equation with a potential $q(x)=\cos x+\alpha \cos (k x+\theta)$ depends very sensitively on the rationality of $k$. Presumably, an appropriately defined quantum ballistic coefficient displays a discontinuous behavior similar to what we have found in the classical case. Due to the generality of the results proven here, similar arbitrarily slow decay results concerning an appropriately defined, time-dependent quantum ballistic coefficient would then follow.

The main ingredient in our analysis is the observation that any (real-valued) function $f(k, t)$ jointly continuous in $k$ and $t$ with $\lim _{t \rightarrow \infty} f(k, t)$ densely discontinuous in $k$ has arbitrarily slow decay for a dense set of $k$ 's. 


\section{GENERAL RESULTS ON APPROACH TO LIMITS}

Let $f(\mathbf{k}, t): \mathbb{R}^{N} \times[0, \infty) \rightarrow \mathbb{R}$ satisfy the following conditions:

(i) $f(\mathbf{k}, t)$ is continuous in $\mathbf{k} \in \mathbb{R}^{N}$ and $t \in[0, \infty)$,

(ii) $\lim _{t \rightarrow \infty} f(\mathbf{k}, t)=F(\mathbf{k})$ exists for all $\mathbf{k} \in \mathbb{R}^{N}$, and

(iii) the set $A \subset \mathbb{R}^{N}$ of k's at which $F$ is discontinuous is dense in $\mathbb{R}^{N}$.

We begin our analysis with the basic observation that given $\mathbf{k} \in A$, there exists a $\mathbf{k}^{\prime} \in A$ such that $F\left(\mathbf{k}^{\prime}\right)$ differs from $F(\mathbf{k})$ by a substantial amount with $\mathbf{k}^{\prime}$ arbitrarily close to $\mathbf{k}$, so that $f\left(\mathbf{k}^{\prime}, t\right)$ can be made as close as we like to $f(\mathbf{k}, t)$ for as long as we like. More precisely,

Lemma 2.1. Let $f(\mathbf{k}, t)$ satisfy (2.1)-(2.3), and let

$$
G(\mathbf{k}, \tau, \varepsilon, \lambda)=\left\{\mathbf{k}^{\prime} \mid \begin{array}{l}
\left|f\left(\mathbf{k}^{\prime}, t\right)-f(\mathbf{k}, t)\right|<\varepsilon, \\
\text { and }\left|F\left(\mathbf{k}^{\prime}\right)-F(\mathbf{k})\right|>\lambda
\end{array} \quad \forall t \leq \tau\right\} .
$$

If $0<\lambda<\varlimsup_{\lim _{\mathbf{k}^{\prime} \rightarrow \mathbf{k}}}\left|F\left(\mathbf{k}^{\prime}\right)-F(\mathbf{k})\right| \equiv \delta(\mathbf{k})$, then

$$
\mathbf{k} \in \overline{G(\mathbf{k}, \tau, \varepsilon, \lambda) \cap A} \text {. }
$$

Proof. Clearly there exists $\mathbf{k}^{\prime} \in G$ arbitrarily close to $\mathbf{k}$. If $\mathbf{k}^{\prime}$ is not also in $A$, then $\mathbf{k}^{\prime}$ is a point of continuity of $F$, and, because of the density of $A$, there is a $\mathbf{k}^{\prime \prime} \in G \cap A$ arbitrarily close to $\mathbf{k}^{\prime}$.

Lemma 2.2. Let $f(\mathbf{k}, t)$ satisfy (2.1)-(2.3), and let $\hat{g}_{n}(t)$ be any sequence of functions with $\hat{g}_{n}(t) \downarrow 0, t \rightarrow \infty$, for all $n=0,1,2, \ldots$. Then the set $\Gamma$ of $\mathbf{k}$ 's for which there exists a sequence $t_{n} \rightarrow \infty$ such that

$$
\left|f\left(\mathbf{k}, t_{n}\right)-F(\mathbf{k})\right|>\hat{g}_{n}\left(t_{n}\right)
$$

is dense in $\mathbb{R}^{N}$.

Proof. Fix any $\mathbf{k}_{0} \in A$ and $\varepsilon^{\prime}>0$. We will show that there exists $\mathbf{k}$ such that (2.6) is satisfied for some sequence $t_{n} \rightarrow \infty$ and $\left|\mathbf{k}-\mathbf{k}_{0}\right|<\varepsilon^{\prime}$.

Let

$$
T(\mathbf{k}, \varepsilon)=\sup \{t \geq 0|| f(\mathbf{k}, t)-F(\mathbf{k}) \mid \geq \varepsilon\}
$$

(if the set on the right is empty, set $T(\mathbf{k}, \varepsilon)=0$ ). Let $\varepsilon_{0}=\delta\left(\mathbf{k}_{0}\right) / 9$, with $\delta\left(\mathbf{k}_{0}\right)$ as in Lemma 2.1 , let

$$
s_{0}=T\left(\mathbf{k}_{0}, \varepsilon_{0}\right)+\inf \left\{t \geq 0 \mid \hat{g}_{0}(t)<\varepsilon_{0}\right\}+1,
$$

and choose $\mathbf{k}_{1} \in G\left(\mathbf{k}_{0}, s_{0}, \varepsilon_{0}, \delta\left(\mathbf{k}_{0}\right)-\varepsilon_{0}\right) \cap A$ satisfying $\left|\mathbf{k}_{1}-\mathbf{k}_{0}\right|<\varepsilon^{\prime} / 2$. Let

Suppose now that $\mathbf{k}_{0}, \mathbf{k}_{1}, \ldots, \mathbf{k}_{n}$ have been chosen, using $\varepsilon_{0}, \ldots, \varepsilon_{n-1}$.

$$
\varepsilon_{n}=\inf \left(\varepsilon_{0}, \ldots, \varepsilon_{n-1}, \delta\left(\mathbf{k}_{n}\right) / 9\right),
$$

(2.10) $s_{n}=s_{n-1}+T\left(\mathbf{k}_{n}, \varepsilon_{n}\right)+\inf \left\{t \geq 0 \mid \hat{g}_{n}(t)<\varepsilon_{n}\right\}+1 \quad(\geq n+1)$, 
and choose $\mathbf{k}_{n+1} \in G\left(\mathbf{k}_{n}, s_{n}, 2^{-n} \varepsilon_{n}, \delta\left(\mathbf{k}_{n}\right)-\varepsilon_{n}\right) \cap A$ satisfying $\left|\mathbf{k}_{n+1}-\mathbf{k}_{n}\right|<$ $\varepsilon^{\prime} / 2^{n+1}$.

Let $\mathbf{k}=\lim _{n \rightarrow \infty} \mathbf{k}_{n}$. Then $\left|\mathbf{k}-\mathbf{k}_{0}\right|<\varepsilon^{\prime}$, and

$$
\begin{aligned}
\left|f\left(\mathbf{k}, s_{n}\right)-F\left(\mathbf{k}_{n}\right)\right| & \leq \sum_{m=n}^{\infty}\left|f\left(\mathbf{k}_{m+1}, s_{n}\right)-f\left(\mathbf{k}_{m}, s_{n}\right)\right|+\left|f\left(\mathbf{k}_{n}, s_{n}\right)-F\left(\mathbf{k}_{n}\right)\right| \\
& <2 \varepsilon_{n}+\varepsilon_{n}=3 \varepsilon_{n},
\end{aligned}
$$

so that

$$
\begin{aligned}
& \left|f\left(\mathbf{k}, s_{n}\right)-f\left(\mathbf{k}, s_{n+1}\right)\right| \\
& \quad \geq\left|F\left(\mathbf{k}_{n+1}\right)-F\left(\mathbf{k}_{n}\right)\right|-\left|f\left(\mathbf{k}, s_{n}\right)-F\left(\mathbf{k}_{n}\right)\right|-f\left|\left(\mathbf{k}, s_{n+1}\right)-F\left(\mathbf{k}_{n+1}\right)\right| \\
& \quad>\left(\delta\left(\mathbf{k}_{n}\right)-\varepsilon_{n}\right)-3 \varepsilon_{n}-3 \varepsilon_{n+1} \geq \delta\left(\mathbf{k}_{n}\right)-7 \varepsilon_{n},
\end{aligned}
$$

and

$$
\left|f\left(\mathbf{k}, t_{n}\right)-F(\mathbf{k})\right|>\frac{\delta\left(\mathbf{k}_{n}\right)-7 \varepsilon_{n}}{2} \geq \varepsilon_{n}>\hat{g}_{n}\left(t_{n}\right),
$$

either for $t_{n}=s_{n}$ or for $t_{n}=s_{n+1}$.

To state the main theorem, we require the following:

Definition. Let $h(t)$ and $g(t)$ be real-valued functions on $[0, \infty)$. We say that $h(t)$ is greater than $g(t)$ infinitely often and write $h(t) \underset{\text { i.o. }}{>} g(t)$ if there exists a sequence $t_{n} \rightarrow \infty$ such that $h\left(t_{n}\right)>g\left(t_{n}\right)$ for all $n$.

Theorem 2.1. Let $f(\mathbf{k}, t)$ satisfy (2.1)-(2.3) and let $\left\{g_{n}(t)\right\}$ be any sequence of functions on $[0, \infty)$ with $g_{n}(t) \rightarrow 0, t \rightarrow \infty$, for all $n$. Then the set $\Gamma$ of $\mathbf{k}$ 's for which

$$
|f(\mathbf{k}, t)-F(\mathbf{k})| \underset{i . o .}{>} g_{n}(t), \quad \forall n
$$

is dense in $\mathbb{R}^{N}$.

Proof. Without loss of generality we may assume that the $g_{n}$ 's are bounded, and, by replacing $g_{n}(t)$ by $\sup _{t^{\prime}>t} g_{n}\left(t^{\prime}\right)$, that $g_{n}(t) \downarrow 0, t \rightarrow \infty$. Then we let $\hat{g}_{n}=\sup \left(g_{0}, g_{1}, \ldots, g_{n}\right)$ and apply Lemma 2.2 .

In order to state a striking consequence of Theorem 2.1 , we utilize the notion of an expressible function, i.e., one which can be defined, either explicitly or implicitly, using standard mathematical symbols. An example of such an implicitly defined function is one that satisfies, say, an explicit differential or integral equation which has a unique solution. Since any expressible function is determined by a finite string of symbols from a finite alphabet, there are only countably many such functions. For example, the reader should note well that most of the functions $f_{\alpha}(x)=\alpha x, \alpha \in \mathbb{R}$, are not expressible - there are only a countable number of expressible reals. (This notion of expressibility depends, of course, upon a fixed choice of "standard mathematical symbols," i.e., upon a choice of formal language.) From Theorem 2.1 we immediately obtain the 
following:

Theorem 2.2. Let $f(\mathbf{k}, t)$ satisfy (2.1)-(2.3). Then the set $\Gamma$ of $\mathbf{k}$ 's for which

$$
|f(\mathbf{k}, t)-F(\mathbf{k})| \underset{i . o .}{>} g(t),
$$

for every expressible function $g(t)$ with $g(t) \rightarrow 0$ as $t \rightarrow \infty$ is dense in $\mathbb{R}^{N}$.

Remark. To appreciate how slowly $|f(\mathbf{k}, t)-F(\mathbf{k})|$ decays when $\mathbf{k} \in \Gamma$, observe that for $\mathbf{k} \in \Gamma,|f(\mathbf{k}, t)-F(\mathbf{k})| \underset{\text { i.o. }}{>}(\log \cdots \log t)^{-1}$, for any fixed number of iterations of the logarithm. Indeed, no law, be it algebraic, logarithmic, or any other, can express such slow decay.

Remark. Theorem 2.2 remains true if $t$ is allowed to approach an endpoint of any interval $(a, b)$ for which the conditions (2.1)-(2.3) are satisfied in the obvious sense.

It is natural to ask about the size of the set $\Gamma$ of $\mathbf{k}$ 's for which $f(\mathbf{k}, t)$ approaches its limit "arbitrarily slowly" as $t \rightarrow \infty$, whose existence and density follow from the above theorems. In all situations where we have been able to check conditions (2.1)-(2.3) for a concretely realized $f(\mathbf{k}, t)$, such as for diffusion in one dimension, $\Gamma$ is presumably of Lebesgue measure zero. However, in these situations, a further condition, stated below, is also satisfied, which implies that $\Gamma$ is nonetheless (topologically) generic.

Theorem 2.3. Suppose $f(\mathbf{k}, t)$ satisfies, in addition to (2.1)-(2.3),

(2.16) (iv) $A^{c}$ is dense, and there exists a continuous function $\varphi(\mathbf{k})$ on $\mathbb{R}^{N}$ such that $F(\mathbf{k})=\varphi(\mathbf{k}) \Leftrightarrow \mathbf{k} \in A^{c}$.

Then for any sequence of functions $g_{n}(t) \downarrow 0, t \rightarrow \infty$, the set $\Gamma$ of $\mathbf{k}$ 's for which

$$
|f(\mathbf{k}, t)-F(\mathbf{k})| \underset{\text { i.o. }}{>} g_{n}(t), \quad \forall n,
$$

contains a dense $\mathscr{G}_{\delta}$.

Proof. Let $G(\mathbf{k}, \tau, \varepsilon, \lambda)$ be as in Lemma 2.1 and for $g(t) \downarrow 0, t \rightarrow \infty$, let

$$
G(g)=\bigcup_{\mathbf{k} \in A} G(\mathbf{k}, t(g, \mathbf{k}), \varepsilon(\mathbf{k}), \bar{\delta}(\mathbf{k})-\varepsilon(\mathbf{k})),
$$

where

$$
\begin{gathered}
\bar{\delta}(\mathbf{k})=\varlimsup_{\substack{\mathbf{k}^{\prime} \rightarrow \mathbf{k} \\
\mathbf{k}^{\prime} \in A^{c}}}\left|F\left(\mathbf{k}^{\prime}\right)-F(\mathbf{k})\right|, \\
\varepsilon(\mathbf{k})=\bar{\delta}(\mathbf{k}) / 4,
\end{gathered}
$$

and

$$
t(g, \mathbf{k})=T(\mathbf{k}, \varepsilon(\mathbf{k}))+\inf \{t \geq 0 \mid g(t)<\varepsilon(\mathbf{k})\}+1,
$$

where $T(\mathbf{k}, \varepsilon)$ is defined in (2.7). By virtue of (2.16),

$$
\mathbf{k} \in \overline{\operatorname{Int}(G(\mathbf{k}, \tau, \varepsilon, \lambda))} \text {, }
$$

provided $0<\lambda<\bar{\delta}(\mathbf{k})$. Therefore $G(g)$ contains a dense open set. 
Given a sequence $g_{n}(t) \downarrow 0$, define

$$
\hat{g}_{n}=\sup \left(g_{1}, \ldots, g_{n}\right) \text {. }
$$

We may assume without loss of generality that $\lim _{n \rightarrow \infty} \hat{g}_{n}(t)=\infty$ for all $t \geq 0$. Let

$$
G=\bigcap_{n} G\left(\hat{g}_{n}\right) .
$$

Then, by the Baire category theorem, $G$ contains a dense $\mathscr{G}_{\delta}$, and by construction $G \subset \Gamma$.

Remark. With condition (2.16), the set $\Gamma$ in Theorem 2.2 also contains a dense $\mathscr{G}_{\delta}$.

\section{DIFFUSION IN QUASIPERIODIC POTENTIALS}

a. Formulation. Let $V(\underline{x}), \underline{x} \in \mathbb{R}^{d}$, be uniformly bounded and smooth, i.e., let it have uniformly bounded derivates to the third order. Given $V(\underline{x})$, we consider the $\mathbb{R}^{d}$-valued process $\underline{X}_{t}$ governed by

$$
d \underline{X}_{t}=-\nabla V\left(\underline{X}_{t}\right) d t+d \underline{W}_{t},
$$

where $\underline{X}_{0}=0$ and $\underline{W}_{t}$ is standard Brownian motion with mean 0 and covariance matrix $t I$, where $I$ is the identity. The transition density $u(\underline{x}, t)$ satisfies the (forward) equation

$$
\frac{\partial u}{\partial t}=L^{*} u, \quad \lim _{t \downarrow 0} u(\underline{x}, t)=\delta(\underline{x}),
$$

where

$$
L^{*}=\frac{1}{2} \Delta+\nabla \cdot(\nabla V \cdot) .
$$

We shall be interested in quasiperiodic $V$, with $n$ frequencies, defined in the following way. Let $\widehat{V}(\underline{\theta})$ be a smooth function on the unit $n$-torus $T^{n}=$ $\mathbb{R}^{n} / \mathbb{Z}^{n}, \underline{\theta} \in T^{n}$, which we identify with the obvious periodic function on $\mathbb{R}^{n}$. The local potential field $V(\underline{x}) \equiv V_{\mathbf{k}}(\underline{x}, \underline{\theta}), \underline{x} \in \mathbb{R}^{d}$, is obtained from $\widehat{V}$ via

$$
V_{\mathbf{k}}(\underline{x}, \underline{\theta})=\widehat{V}(\underline{\theta}+\mathbf{k} \underline{x})=\widehat{V}\left(\tau_{\underline{x}}^{\mathbf{k}} \underline{\theta}\right),
$$

with translations on $\mathbb{R}^{n}$ given by

$$
\tau_{\underline{x}}^{\mathbf{k}} \underline{\theta}=\underline{\theta}+\mathbf{k} \underline{x}=\underline{\theta}+\sum_{i=1}^{d} \underline{k}_{i} x_{i},
$$

where $\mathbf{k}$ is an $n$-by $d$-matrix $\mathbf{k}=\left[\underline{k}_{1}^{T}, \ldots, \underline{k}_{d}^{T}\right], \underline{k}_{i} \cdot \underline{k}_{j}=0, i \neq j, \underline{k}_{i} \in \mathbb{R}^{n}$.

The flow on $T^{n}$ defined by (3.5) leaves invariant Lebesgue measure $d \underline{\theta}$ on $T^{n}$. It is also ergodic relative to $d \underline{\theta}$ when the equations $\underline{k}_{1} \cdot \underline{j}=0, \ldots, \underline{k}_{d} \cdot \underline{j}=0$ have no simultaneous integral solutions $\underline{j} \in \mathbb{Z}^{n}, \underline{j} \neq 0$ [14]. We say that $\mathbf{k}$ is "irrational" in this case, i.e., when $\tau_{\underline{x}}^{\mathbf{k}}$ is ergodic, and is "rational" otherwise. 
When $n=2, d=1$, and $\mathbf{k}=\underline{k}=\left[k_{1}, k_{2}\right]^{T}, \mathbf{k}$ is "irrational" when $k_{2} / k_{1}$ is irrational. When $n>d+1, \mathbf{k}$ can have various degrees of rationality, depending on the dimension of the ergodic components of $\tau_{\underline{x}}^{\mathbf{k}}$.

For $\underline{X}_{t}$ the process governed by (3.1) with $V=V_{k}(\underline{x}, \underline{\theta}), \varepsilon \underline{X}_{t / \varepsilon^{2}}$ converges [1-3] as $\varepsilon \rightarrow 0$ to $\underline{W}_{t}\left(\mathbf{D}^{*}(\mathbf{k})\right)$, with $\mathbf{D}^{*}(\mathbf{k})=\lim _{t \rightarrow \infty} \mathbf{D}(\mathbf{k}, t), D_{i j}(\mathbf{k}, t)=$ $E\left[X_{t}^{i} X_{t}^{j}\right] / t$, where $E$ denotes expectation over Brownian motion paths in (3.1) as well as an average over $T^{n}$ with respect to the equilibrium measure

$$
\mu(d \underline{\theta})=e^{-2 \widehat{V}(\underline{\theta})} d \underline{\theta} / \int_{T^{n}} e^{-2 \widehat{V}(\underline{\theta})} d \underline{\theta} .
$$

Let $\mathscr{D}(k, t)=\operatorname{tr}\left(\mathbf{D}^{*}(\mathbf{k}, t)\right)$, and let $D^{*}(\mathbf{k})=\operatorname{tr}\left(\mathbf{D}^{*}(\mathbf{k})\right)$. It follows easily from $(3.1)[1,11]$

$$
D^{*}(\mathbf{k})=1-\int_{0}^{\infty} c(t) d t
$$

where $c(t)=E\left[\nabla V\left(\underline{X}_{0}\right) \cdot \nabla V\left(\underline{X}_{s}\right)\right] \geq 0$, and that

$$
\mathscr{D}(\mathbf{k}, t)=D^{*}(\mathbf{k})+\frac{1}{t} \int_{0}^{t} d s \int_{s}^{\infty} c(u) d u .
$$

b. Long time/low frequency asymptotics. In order to apply the results of $\S 2$ to $\mathscr{D}(\mathbf{k}, t)$, we need only discuss the discontinuous behavior of $D^{*}(\mathbf{k})$, as the continuity of $\mathscr{D}(\mathbf{k}, t)$ in $\mathbf{k}$ and $t$ is routine. In one dimension, there is an exact formula (see, e.g., [5]) for $D^{*}$,

$$
D^{*}(\underline{k})=\int_{T^{n}}\left[\left\langle e^{2 \widehat{V}}\right\rangle_{\underline{k}}\left\langle e^{-2 \widehat{V}}\right\rangle_{\underline{k}}\right]^{-1} \mu(d \theta)
$$

where $\langle\cdot\rangle_{\underline{k}}$ denotes averaging over a trajectory of the flow $\underline{\dot{\theta}}=\underline{k}$, which is ergodic only when $\underline{k}$ is irrational. In this case, $\langle\cdot\rangle_{\underline{k}}$ amounts to integration over all of $T^{n}$. However, when $\underline{k}$ is rational, the trajectory degenerates to a closed orbit, over which the integration is different from its value over all of $T^{n}$. Thus [5] there is typically a dense set of rationals on which $D^{*}(k)$ is discontinuous, in which case $D^{*}(k)$ in fact satisfies condition (2.16), with $\varphi(k)=\bar{D}$, the common value of $D^{*}(k)$ for irrational $k$.

While there is no such general argument in higher dimensions, where an explicit formula for $\mathbf{D}^{*}(\mathbf{k})$ does not, to our knowledge, exist, the integrals involved in representation formulas for $\mathbf{D}^{*}$ involve averages over trajectories similar to $\langle\cdot\rangle_{\mathbf{k}}$ above, and similarly discontinuous. Thus we believe that, as in one dimension, there should typically be a dense set of $\mathbf{k}$ 's at which $\mathbf{D}^{*}(\mathbf{k})$ is discontinuous [5] (see also [15] for a concrete example). Accordingly, we shall state our results for systems with this property and state the following definition:

Definition 3.1. A potential $\widehat{V}$ on $T^{n}$ is typical if $D^{*}(\mathbf{k})$ is discontinuous in $\mathbf{k}$ on a dense set in $\mathbb{R}^{N}$. 
Now as an immediate consequence of Theorem 2.2, we have the following: Theorem 3.1. Let $\widehat{V}$ on $T^{n}$ be typical. Then for diffusion $\underline{X}_{t}$ in $\mathbb{R}^{d}$ satisfying (3.1) with $V_{\mathbf{k}}(\underline{x}, \theta)=\hat{V}(\underline{\theta}+\mathbf{k} \underline{x}), \underline{x} \in \mathbb{R}^{d}$, and $\underline{\theta} \in T^{n}$, the set $\Gamma$ of $\mathbf{k}$ 's for which

$$
\left|\mathscr{D}(\mathbf{k}, t)-D^{*}(\mathbf{k})\right| \underset{\text { i.o. }}{>} g(t),
$$

for every expressible function $g(t)$ with $\lim _{t \rightarrow \infty} g(t)=0$, is dense (in $\mathbb{R}^{n d}$ ).

Remark. In one dimension, and presumably in higher as well, the set $\Gamma$ in Theorem 3.1 contains a dense $\mathscr{G}_{\delta}$ set.

As another immediate consequence of Theorem 2.2 (with $t=1 / \omega$ ), we state the corresponding results about the frequency dependent diffusivity

$$
\widetilde{D}(\mathbf{k}, \omega)=\omega^{2} \int_{0}^{\infty} e^{-\omega t} E\left[\underline{X}_{t}^{2}\right] d t,
$$

which can also be written in terms of the velocity autocorrelation function $c(t)$,

$$
\widetilde{D}(\mathbf{k}, \omega)=1-\int_{0}^{\infty} e^{-\omega t} c(t) d t
$$

We note that $\lim _{\omega \rightarrow 0} \tilde{D}(\mathbf{k}, \omega)=D^{*}(\mathbf{k})$.

Theorem 3.2. Let $\widehat{V}$ on $T^{n}$ be typical with $\underline{X}_{t} \in \mathbb{R}^{d}$ as in Theorem 3.1. Then the set of $\mathbf{k}$ 's for which

$$
\left|\widetilde{D}(\mathbf{k}, \omega)-D^{*}(\mathbf{k})\right| \underset{i .0 .}{>} g(\omega), \quad \omega \rightarrow 0,
$$

for every expressible function $g(\omega)$ with $\lim _{\omega \rightarrow 0} g(\omega)=0$ is dense.

From Theorem 3.1 we can prove

Theorem 3.3. Let $\widehat{V}$ on $T^{n}$ be typical with $\underline{X}_{t} \in \mathbb{R}^{d}$, as in Theorem 3.1. Then the set $\widetilde{\Gamma}$ of $\mathbf{k}$ 's for which

$$
c_{\mathbf{k}}(t) \underset{\text { i.o. }}{>} h(t), \quad t \rightarrow \infty,
$$

for every expressible function $h(t)$, which is integrable on $[0, \infty)$, is dense.

Proof. Fix $\mathbf{k}$, and let $\psi(\mathbf{k}, t)=\left|\mathscr{D}(\mathbf{k}, t)-D^{*}(\mathbf{k})\right|$. Suppose there is an expressible function $\tilde{h}(t)$ integrable on $[0, \infty)$ such that

$$
c(t) \leq \tilde{h}(t), \quad \forall t \geq T \geq 0 .
$$

Then there is an expressible function $h(t)$ integrable on $[0, \infty)$ such that $c(t) \leq$ $h(t) \forall t \in[0, \infty)$, so that

$$
\frac{1}{t} \int_{0}^{t} d s \int_{s}^{\infty} c(u) d u \leq \frac{1}{t} \int_{0}^{t} d s \int_{s}^{\infty} h(u) d u .
$$

By (3.8),

$$
\psi(\mathbf{k}, t) \leq g(t)=\frac{1}{t} \int_{0}^{t} d s \int_{s}^{\infty} h(u) d u, \quad \forall t \geq 0
$$


Since $h$ is expressible and integrable, $g(t)$ is expressible with $g(t) \rightarrow 0$ as $t \rightarrow \infty$, so that $\mathbf{k}$ is not in $\Gamma$, the dense set described in Theorem 3.1. Thus $\Gamma \subset \widetilde{\Gamma}$.

In order to state our results about the spectral measure, we introduce the environment process $\underline{\theta}_{t}=\tau_{\underline{X}_{t}}^{\mathbf{k}} \underline{\theta}\left(\bmod \mathbf{Z}^{n}\right)$, which determines the potential field seen by the particle at time $t$. This process is reversible with respect to the equilibrium measure $\mu(d \underline{\theta})$ and is generated by $\widehat{L}_{\mathbf{k}}=\frac{1}{2} \Delta_{\mathbf{k}}-\nabla_{\mathbf{k}} V \cdot \nabla_{\mathbf{k}}$, where $\nabla_{\mathbf{k}}$ is gradient on $T^{n}$ arising from the flow $\tau_{\underline{x}}^{\mathbf{k}}$. $\widehat{L}_{\mathbf{k}}$ is selfadjoint in $L^{2}\left(T^{n}, d \mu\right)$ and has negative spectrum in $(-\infty, 0]$ with a family of projection-valued measures $P_{\lambda}$ on $(-\infty, 0] .\left(\widehat{L}_{\mathbf{k}}\right.$ is unitarily equivalent to $H_{\mathbf{k}}=\frac{1}{2} \Delta_{\mathbf{k}}+q$ on $L^{2}\left(T^{n}, d \underline{\theta}\right)$ with $q=\frac{1}{2}\left(\nabla_{\mathbf{k}} V \cdot \nabla_{\mathbf{k}} V-\Delta_{\mathbf{k}} V\right)$, via $e^{\widehat{V}} H e^{-\widehat{V}}=\widehat{L}$.) We consider the particular spectral measure $\nu$ of $P_{\lambda}$ associated with $\nabla_{\mathbf{k}} V, \nu=\left\langle\nabla_{\mathbf{k}} V \cdot P_{\lambda} \nabla_{\mathbf{k}} V\right\rangle$, where $\langle\cdot\rangle$ here means integration over $T^{n}$ with respect to $\mu$. Using the semigroup $\exp \left(\widehat{L}_{\mathbf{k}} t\right)$ one can write

$$
c(t)=\int_{-\infty}^{0} e^{\lambda t} d \nu(\lambda)
$$

Now we can state

Theorem 3.4. Let $\widehat{V}$ on $T^{n}$ be typical with $\underline{X}_{t} \in \mathbb{R}^{d}$ as in Theorem 3.1. Then the set $\Gamma^{*}$ of $\mathbf{k}$ 's for which

$$
\nu_{\mathbf{k}}(d \lambda) \underset{\text { i.o. }}{>} \eta(d \lambda), \quad \lambda \rightarrow 0^{-},
$$

for every expressible measure $\eta$ on $(-\infty, 0)$ with $\int_{-\infty}^{0} \eta(d \lambda) /|\lambda|<\infty$, is dense.

(By $\nu(d \lambda) \underset{\text { i.o. }}{>} \eta(d \lambda), \lambda \rightarrow 0^{-}$, we mean that there is a sequence of intervals $\left(t_{n}, s_{n}\right) \subset(-\infty, 0), t_{n} \rightarrow 0$, such that $\nu\left(t_{n}, s_{n}\right)>\eta\left(t_{n}, s_{n}\right), \forall n$.)

Proof. Fix k. Suppose there is an expressible measure $\eta(d \lambda)$ with

$$
\int_{-\infty}^{0} \eta(d \lambda) /|\lambda|<\infty
$$

such that

$$
\nu \leq \eta
$$

on $[\Lambda, 0)$ for some $\Lambda<0$. Then

$$
c(t)=\int_{-\infty}^{0} e^{\lambda t} d \nu(\lambda) \leq e^{\Lambda t} \nu(-\infty, \Lambda]+\int_{\Lambda}^{0} e^{\lambda t} d \eta(\lambda) .
$$

Thus, since $\nu(-\infty, 0]<\infty$ and $\int_{-\infty}^{0} \frac{\eta(d \lambda)}{|\lambda|}<\infty$, there exists an expressible, integrable function $h(t)$ such that $c(t) \leq h(t), \forall t \geq 0$. Therefore $\mathbf{k} \notin \widetilde{\Gamma}$ (of Theorem 3.3); i.e., $\widetilde{\Gamma} \subset \Gamma^{*}$. 


\section{CONDUCTION IN QUASIPERIODIC MEDIA}

a. Formulation. Let $\hat{\sigma}(\underline{\theta})$ be a smooth function on $T^{n}$. Analogous to $V_{\mathbf{k}}$, we define $\sigma_{\mathbf{k}}(\underline{x}, \theta)=\hat{\sigma}(\underline{\theta}+\mathbf{k} \underline{x})$. Given $\sigma_{\mathbf{k}}$ on $\mathbb{R}^{d}$, we take a finite cubic sample $C_{L}=\left\{\underline{x}:-L \leq x_{i} \leq L, i=1, \ldots, d\right\}$ of side $2 L$ centered at the origin. Let $u_{L}$ be the solution of

$$
\begin{gathered}
\nabla \cdot\left(\sigma_{\mathbf{k}} \nabla u_{L}\right)=0, \quad \underline{x} \in C_{L}, \\
\frac{\partial u_{L}}{\partial x_{i}}=0 \text { on } x_{i}=L \quad \text { or } x_{i}=-L, \quad i \neq 1, \quad-L \leq x_{1} \leq L, \\
u_{L}=-L \text { on } x_{1}=-L, \quad-L \leq x_{i} \leq L, \quad i \neq 1, \\
u_{L}=L \text { on } x_{1}=L, \quad-L \leq x_{i} \leq L, \quad i \neq 1 .
\end{gathered}
$$

Then define $\sigma^{*}(\mathbf{k}, L, \underline{\theta})$ by

$$
\sigma^{*}(\mathbf{k}, L, \underline{\theta}) \underline{e}_{1}=\frac{1}{(2 L)^{d}} \int_{C_{L}} \sigma_{\mathbf{k}}(\underline{x}, \underline{\theta}) \nabla u_{L} d \underline{x} .
$$

We shall be interested in averaging $\sigma^{*}(\mathbf{k}, L, \underline{\theta})$ over $T^{n}$ to obtain

$$
\sigma^{*}(\mathbf{k}, L)=\int_{T^{n}} \sigma^{*}(\mathbf{k}, L, \underline{\theta}) d \underline{\theta},
$$

which is continuous in $\mathbf{k}$, as well as $L$. It is easily obtained from [4, Appendix] that

$$
\lim _{L \rightarrow \infty} \sigma^{*}(\mathbf{k}, L)=\sigma^{*}(\mathbf{k}),
$$

where $\sigma^{*}(\mathbf{k})$ is the effective conductivity of the medium $\sigma_{\mathbf{k}}(\underline{x}, \underline{\theta})$. (See [4] for the precise definition of $\sigma^{*}$.)

b. Large sample size asymptotics. As in $\S 3$, we say that $\hat{\sigma}$ on $T^{n}$ is "typical" if $\sigma^{*}(\mathbf{k})$ is discontinuous in $\mathbf{k}$ on a dense subset of $\mathbb{R}^{N}$. We have again as an immediate consequence of Theorem 2.1 the following theorem:

Theorem 4.1. Let $\hat{\sigma}$ on $T^{n}$ be typical. Then for conduction satisfying (4.1)-(4.5) in a cubic sample $C_{L}$ of a medium with local conductivity $\sigma_{\mathbf{k}}(\underline{x}, \underline{\theta})=\hat{\sigma}(\underline{\theta}+\mathbf{k} \underline{x})$, the set $\Gamma$ of k's for which

$$
\left|\sigma^{*}(\mathbf{k}, L)-\sigma^{*}(\mathbf{k})\right|>\text { i.o. } g(L), \quad L \rightarrow \infty,
$$

for every expressible function $g(L)$ with $\lim _{L \rightarrow \infty} g(L)=0$, is dense.

Remark. In one dimension, and presumably in higher dimensions, the set $\Gamma$ in Theorem 4.1 contains a dense $\mathscr{G}_{\delta}$.

\section{REFERENCES}

1. A. DeMasi, P. Ferrari, S. Goldstein, and D. W. Wick, J. Statist. Phys. 55 (1989), 787.

2. G. Papanicolaou and S. Varadhan, Random fields (Colloquia Mathematica Societatis János Bolyai 27), J. Fritz, J. L. Lebowitz, and D. Szász, eds., North-Holland, Amsterdam, 1982. 
3. S. M. Kozlov, Soviet Math. Dokl. 18 (1977), 1323.

4. K. Golden and G. Papanicolaou, Comm. Math. Phys. 90 (1983), 473.

5. K. Golden, S. Goldstein, and J. L. Lebowitz, Phys. Rev. Lett. 55 (1985), 2692.

6. B. J. Adler and T. E. Wainwright, Phys. Rev. A1 (1970), p. 18.

7. Ya. G. Sinai, Ann. N.Y. Acad. Sci. 357 (1980), 143.

8. H. van Beijeren and H. Spohn, J. Statist. Phys. 31 (1983), 231.

9. M. H. Ernst, J. Machta, J. R. Dorfman, and H. van Beijeren, J. Statist. Phys. 34 (1984), 477.

10. K. Golden, S. Goldstein, and J. L. Lebowitz, Ann. Probab. 16 (1988), 1127.

11. _ J. Statist. Phys. 51 (1988), 637.

12. B. Simon, Adv. in Appl. Math. 3 (1982), 463.

13. T. Spencer, Statphys. 16 (H. E. Stanley, ed.), North-Holland, Amsterdam, 1986.

14. M. Reed and B. Simon, Methods of modern mathematical physics, vol. I: functional analysis, Academic Press, New York, 1980.

15. K. Golden, S. Goldstein, ard J. L. Lebowitz, Discontinuous behavior of effective transport coefficients in quasiperiodic media, J. Statist. Phys. 58 (1990), 669.

Department of Mathematics, Princeton University, Princeton, New Jersey 08544

Department of Mathematics, Rutgers University, New Brunswick, New Jersey 08903 